\title{
Optical spectropolarimetry with incomplete data sets (Research Note)
}

\author{
J. R. Maund \\ Department of Astronomy and McDonald Observatory, The University of Texas at Austin, 1 University Station, C1400, Austin, \\ Texas 78712-0259, USA \\ e-mail: jrm@astro.as.utexas.edu
}

Received 17 July 2007 / Accepted 29 January 2008

\begin{abstract}
Context. Linear spectropolarimetry is a "photon-hungry" observing technique, requiring a specific sequence of observations to determine the Stokes $Q$ and $U$ parameters. For dual-beam spectropolarimeters, the $Q$ and $U$ Stokes parameters can be ideally determined using observations at $N=2$ retarder plate positions. The additional polarization signal introduced by instrumental effects requires the redundancy of $N=4$ observations to correct for these effects and to accurately measure the linear polarization of astronomical objects.

Aims. We wish to determine if the "instrumental signature corrections" for the Stokes $Q$ and $U$ parameters, $\epsilon_{Q}$ and $\epsilon_{U}$, are identical for observations with dual-beam spectropolarimeters. For instances when observations were only acquired at $N=3$ retarder plate angles, we wish to determine if the complete measurement of one Stokes parameter and the associated instrumental signature correction can be used to determine the other Stokes parameter.

Methods. We constructed analytical and Monte Carlo models of a general dual-beam spectropolarimeter to study the factors affecting the assumption $\epsilon_{Q}=\epsilon_{U}$ and the uncertainty thereon. We compared these models with VLT FORS1 linear spectropolarimetry observations.

Results. We find that, in general, $\epsilon_{Q}-\epsilon_{U} \approx 0$, with the variance around zero $\left(\Delta\left(\epsilon_{Q}-\epsilon_{U}\right)\right)$ being directly related to the signal-to-noise ratio of the observations. Observations of a polarized standard star, observed under identical instrumental conditions over the period of 2002-2007, show that the assumption of $\epsilon_{Q}-\epsilon_{U}=0$ is generally true over a long period, although the absolute values of $\epsilon_{Q}$ and $\epsilon_{U}$ vary between observational epochs. While the variance of $\epsilon_{Q}-\epsilon_{U}$ is not dependent on the polarization angle, significant deviations from $\epsilon_{Q}-\epsilon_{U}=0$ arise when $p \gtrsim 20 \%$.

Conclusions. Incomplete VLT FORS1 spectropolarimetry datasets, for which observations at only $N=3$ retarder plate position angles have been acquired, can be analyzed under the assumption that $\epsilon_{Q} \approx \epsilon_{U}$. The uncertainty associated with this assumption is directly related to the signal-to-noise ratio of the observations. This property of the analysis of spectropolarimetry, with dual beam spectropolarimeters, can be used to test for the presence of artifacts affecting individual observations and to assess the quality of the data reduction, when observations at all four retarder plate angles have been acquired.
\end{abstract}

Key words. instrumentation: polarimeters - polarization

\section{Introduction}

Spectropolarimetry is a "photon-hungry" observational technique, requiring a particular sequence of observations to completely and independently determine the polarization properties of astronomical objects. In order to measure particularly low levels of polarization $(\lessgtr 0.1 \%)$, high signal-to-noise ratio $(S / N)$ observations are required at each step in the observing sequence.

For dual-beam spectropolarimeters, such as the European Southern Observatory (ESO) Very Large Telescope (VLT) FOcal Reducer and low dispersion Spectrograph (FORS1; Appenzeller et al. 1998), the determination of the linear polarization Stokes parameters, $Q$ and $U$, requires $N=4$ separate observations. This observing sequence introduces redundancies, but permits the complete determination and correction for instrumental effects that would, if uncorrected for $N=2$ observations, lead to spurious observed polarization.

Target of Opportunity spectropolarimetry (where observations are conducted at specific epochs to study transient events which occur without prior warning) of time-variable phenomena is at a disadvantage, since at each epoch the technique requires a factor $\sim 8$ more time on target than for pure spectroscopy to achieve the same $S / N$ in the individual spectra. For objects such as $\mathrm{SNe}$, the position of the target on the sky may not permit sufficient time on target to conduct all of the necessary observations, leaving the dataset acquired incomplete. If one observation, for one retarder plate angle, is absent, only one Stokes parameter can be independently measured. There is, however, potential redundancy between the observations for the completely determined Stokes parameter and the partially determined parameter such that instrumental effects can be removed, allowing for complete determination of both parameters, but at a higher degree of uncertainty.

Leonard et al. (2001) present a technique where, by assuming the same ratio of gains for the ordinary and extraordinary rays, instrumental effects can be removed to calculate the second Stokes parameter for incomplete datasets. When "normalized flux differences" (e.g. Jehin et al. 2005; Patat \& Romaniello 2006) are used to calculate the Stokes parameter, the consideration is of a correction for the instrumental signature, which quantifies the deviation of data from the ideal case of $N=2$.

Here we present a technique to determine the instrumental signature corrections for observed data, such that, in the event of 
an incomplete observation, both Stokes parameters can be determined. The concept of spectropolarimetry with dual beam spectropolarimeters is, briefly, outlined along with discussion of correction for instrumental effects in Sect. 2. In Sect. 3, analytical and Monte-Carlo models of a dual beam spectropolarimeter are presented, and the effects of various factors on the final measured polarization for complete and incomplete datasets are presented. Real observations of linearly polarized point sources, acquired using VLT FORS1, and the instrumental signature corrections are presented in Sect. 4.

\section{Basic concept and theory}

Dual-beam spectropolarimeters, such as the FORS1 instrument (Appenzeller et al. 1998), use a sequence of a retarder plate and a Wollaston prism to measure the polarization components. Rotation of the retarder plate varies the angle at which the orthogonal polarization components are sampled. The Wollaston prism separates these two components spatially into the ordinary (o) and extraordinary rays (e). At a general retarder plate position $i$, the measured normalized flux difference $F_{i}^{\mathrm{m}}$ is defined (e.g. Jehin et al. 2005) as

$F_{i}^{\mathrm{m}}=\frac{f_{\mathrm{o}}^{i}-f_{\mathrm{e}}^{i}}{f_{\mathrm{o}}^{i}+f_{\mathrm{e}}^{i}}$

such that the total intensity $I=f_{\mathrm{o}}+f_{\mathrm{e}} . F_{i}^{\mathrm{m}}$ is normalized by the total flux intensity and is independent of varying sky transparency and exposure times.

The optimum observing sequence is for a half-wavelength retarder plate to be positioned at $N=4$ position angles $\theta_{i}: \theta_{0}=$ $0.0, \theta_{1}=22^{\circ} .5, \theta_{2}=45^{\circ} .0$ and $\theta_{3}=67.5$.

At $\theta_{0}$, the orthogonal polarization components are in the horizontal and vertical directions. For $\theta_{2}$ the same components are observed, but the beams are swapped such that $f_{\mathrm{o}}^{i}=f_{\mathrm{e}}^{i+2}$. Similarly, for $\theta_{1}$ and $\theta_{3}$ the diagonal polarization components are observed, and the polarization components observed as the ordinary and extraordinary rays are switched between the two observations. The normalized Stokes parameters are given, in terms of normalized flux differences, by Jehin et al. (2005) and Patat \& Romaniello (2006), as

$q=\frac{1}{2}\left(F_{0}^{\mathrm{m}}-F_{2}^{\mathrm{m}}\right)=\frac{1}{2} \sum_{i=0}^{3}\left(F_{i}^{\mathrm{m}}\right) \cos \left(\frac{\pi}{2} i\right)$

$u=\frac{1}{2}\left(F_{1}^{\mathrm{m}}-F_{3}^{\mathrm{m}}\right)=\frac{1}{2} \sum_{i=0}^{3}\left(F_{i}^{\mathrm{m}}\right) \sin \left(\frac{\pi}{2} i\right)$

where the flux Stokes parameters are given by $Q=q I$ and $U=u I$. The redundancy in $N=4$ observations permits the removal of the instrumental effects that differ between the o and e rays. These differences manifest themselves as spurious polarization or depolarization. These instrumental effects are discussed by Patat \& Romaniello (2006). A gain difference between the o and e rays for $N=2$ observations would manifest itself as significant polarization. For spectropolarimetry, flatfields are acquired with the full polarization optics in place, such that the observed flatfields, themselves produced by scattered light, are polarized. In addition, the optical components following the analyzer, such as grisms, filters and lenses, can also act act as linear polarizers, producing a constant additive polarization component which is larger than effects due improper flatfielding using unpolarized flats (Patat \& Romaniello 2006). Importantly,
Patat \& Romaniello also identify a non-additive polarization term, which can arise from a non-ideal Wollaston prism; for most modern dual-beam spectropolarimeters, such as FORS1, the imperfections of the Wollaston prism and the associated effects are negligible. In the case of $N=3$ observations, the instrumental polarization component cannot be removed from the Stokes parameter for which there was only a single observation.

At each retarder plate angle, the measured value of the normalized flux difference can be considered as the sum of the ideal normalized flux difference $\left(F_{i}\right)$ and the instrumental signature correction $\epsilon: F_{i}^{\mathrm{m}}=F_{i}+\epsilon$, such that under ideal conditions $(\epsilon=0)$ a Stokes parameter can be measured using only one value of $F$ at only one retarder plate position (such that the $q$ and $u$ parameters are completely determined with $N=2$ observations).

In the same form as Eqs. (2) and (3), the instrumental signature corrections for the $q$ and $u$ Stokes parameters are, therefore, given by

$$
\begin{aligned}
& \epsilon_{Q}=\frac{1}{2}\left(F_{0}^{\mathrm{m}}+F_{2}^{\mathrm{m}}\right)=\frac{1}{2} \sum_{i=0}^{3} F_{i}^{\mathrm{m}} \cos ^{2}\left(\frac{\pi}{2} i\right) \\
& \epsilon_{U}=\frac{1}{2}\left(F_{1}^{\mathrm{m}}+F_{3}^{\mathrm{m}}\right)=\frac{1}{2} \sum_{i=0}^{3} F_{i}^{\mathrm{m}} \sin ^{2}\left(\frac{\pi}{2} i\right) .
\end{aligned}
$$

Another benefit of this formalism is that the instrumental signature corrections are flux normalized (such that $\epsilon_{Q}$ and $\epsilon_{U}$ are percentages of the total flux) and are independent of the same factors as the normalized flux differences.

In the observing sequence, the primary change between each exposure is the rotation of the retarder plate. For spectropolarimetry of a point source at the centre of the field, the only change should be the orientation of the retarder plate, with the location of the source on the retarder plate unchanged ${ }^{1}$. In this case, therefore, the values of the corrections for the $Q$ and $U$ Stokes parameters should be approximately identical. For an observing sequence with $N=3$, the normalized Stokes parameter with incomplete observations can be determined from the correction determined for the other completely determined parameter, assuming $\epsilon_{Q}=\epsilon_{U}$, as

$$
\begin{aligned}
& q \approx F_{0}^{\mathrm{m}}-\epsilon_{U} \approx-\left(F_{2}^{\mathrm{m}}-\epsilon_{U}\right) \\
& u \approx F_{1}^{\mathrm{m}}-\epsilon_{Q} \approx-\left(F_{3}^{\mathrm{m}}-\epsilon_{Q}\right) .
\end{aligned}
$$

The change in sign of the instrumental signature correction and $F$ is due to switch of the polarization components observed as the o and e rays.

\section{Models}

\subsection{Analytical model}

If the principal difference between the measured o and e rays is purely due to sensitivity, such that the difference can be expressed as a ratio of the gains between the o and e rays $g_{\mathrm{o}} / g_{\mathrm{e}}=g$, then in terms of the ideal values of $f_{\mathrm{o}}$ and $f_{\mathrm{e}}$ (with no instrumental effects) Eq. (1) becomes

$$
F_{i}^{\mathrm{m}}=\frac{g f_{\mathrm{o}}^{i}-f_{\mathrm{e}}^{i}}{g f_{\mathrm{o}}^{i}+f_{\mathrm{e}}^{i}}
$$

${ }^{1}$ If the source is not at the centre of the field passing the beam through different points in the retarder plate, as the retarder plate is rotated, may become important. 


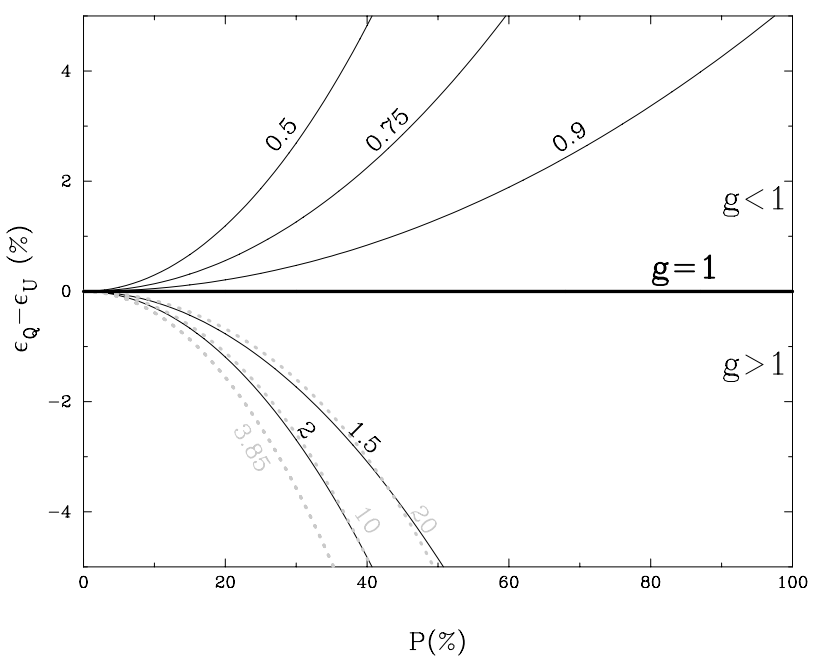

Fig. 1. The analytic form of the dependence of the instrumental signature correction on the gain ratio between the o and e rays and the total polarization, for the case of $p=q$ and $u=0$. For the opposite case, $p=u$ and $q=0$, the top and bottom regions of the diagram are switched. For $g>3.85 \epsilon_{Q}-\epsilon_{U}$ is shown as grey dotted lines.

This form assumes that there are no other effects, such as changing sky transparency (which can become important for very large or small values of $g$ when the flux in only one ray is effectively being measured) or significant phase shift induced by the half-wavelength retarder plate. For $g \neq 1$ the observed intensity changes at each retarder plate angle, depending on the flux and gain for each ray. The ideal values of $f_{\mathrm{o}}$ and $f_{\mathrm{e}}$ (without instrumental effects) are given as

$f_{\mathrm{o}}^{0}=\frac{1}{2} \bar{I}(1+q)=f_{\mathrm{e}}^{2} ; f_{\mathrm{e}}^{0}=\frac{1}{2} \bar{I}(1-q)=f_{\mathrm{o}}^{2}$
$f_{\mathrm{o}}^{1}=\frac{1}{2} \bar{I}(1+u)=f_{\mathrm{e}}^{3} ; f_{\mathrm{e}}^{1}=\frac{1}{2} \bar{I}(1-u)=f_{\mathrm{o}}^{3}$

where the ideal intensity $\bar{I}=f_{\mathrm{o}}+f_{\mathrm{e}}$. Using Eqs. (8)-(10) in Eqs. (4) and (5) gives

$\epsilon_{Q}=\frac{\left(g^{2}-1\right)\left(1-q^{2}\right)}{4 g+(g-1)^{2}\left(1-q^{2}\right)} ; \epsilon_{U}=\frac{\left(g^{2}-1\right)\left(1-u^{2}\right)}{4 g+(g-1)^{2}\left(1-u^{2}\right)}$.

These equations show that the instrumental signature corrections are dependent on the ratio of the gains of the two rays, the total degree of polarization (see Fig. 1) and the polarization angle. In the ideal case, for $g=1$, Eqs. (11) give $\epsilon_{Q}=\epsilon_{U}=0$. The sign of the corrections are dependent on the gain ratio, with

$\epsilon_{Q}(g)-\epsilon_{U}(g)=-\left(\epsilon_{Q}(1 / g)-\epsilon_{U}(1 / g)\right)$.

The magnitude of $\epsilon_{Q}-\epsilon_{U}$ increases for $g>1$, but reaches a stationary point at $g=3.85$. For $g>3.85$ the magnitude of $\epsilon_{Q}-\epsilon_{U}$ decreases due to the predominance of the signal from one ray over the other. This model, therefore, has limited application for realistic observing conditions, for variable observing conditions between observations at different retarder plate angles. For $0.5<g<2$ and $p \lesssim 20 \%$ the magnitude of this deviation, even when only one Stokes parameter is non-zero, is sufficiently small to be within reasonable measurement uncertainties, while the assumption that $\epsilon_{Q}-\epsilon_{U}=0$ can be used to identify if high polarizations are indeed present. The dependence of $\epsilon_{Q}-\epsilon_{U}$ on the polarization angle is shown as Fig. 2. The largest differences

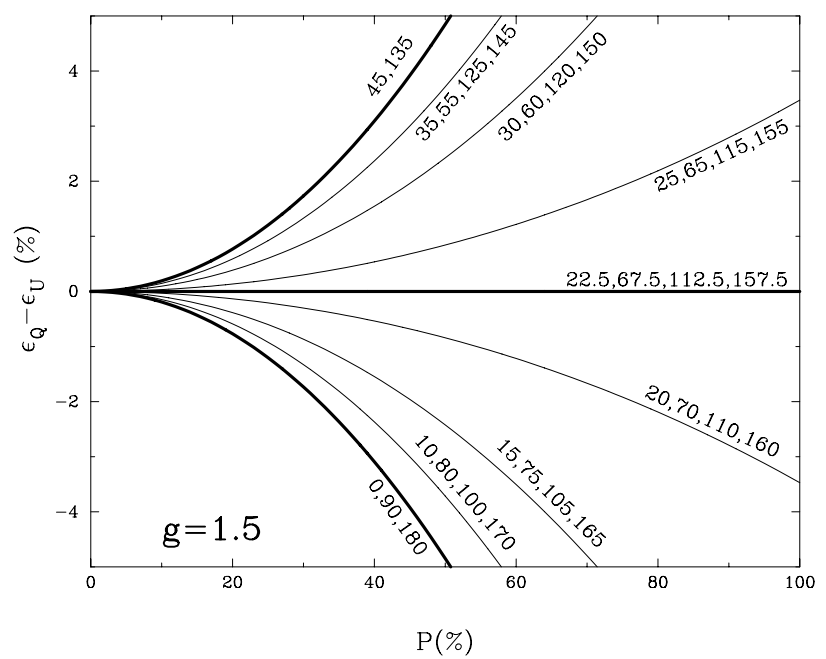

Fig. 2. The difference of the instrumental signature corrections for varying polarization angles, for a fixed gain ratio $g=1.5$.

are measured for either pure $q$ or $u$ components, with mixtures of $q$ and $u$ leading to smaller differences. The instrumental signature corrections are identical, regardless of total polarization, for $q=u, \theta=22.5$. The difference of the instrumental signature corrections is periodic over $90^{\circ}$. For constant polarization and gain, the dependence on polarization angle is characterized by

$\epsilon_{Q}-\epsilon_{U}=\epsilon_{Q}\left(0^{\circ}, p, g\right) \cos (4 \theta)$

where $\epsilon_{Q}\left(0^{\circ}, p, g\right)$ is the $Q$ instrumental signature correction for $\theta=0^{\circ}$ and $q=p$.

\subsection{Monte-Carlo simulations}

In order to test the importance of various realistic instrumental effects, a Monte-Carlo model of a dual beam spectropolarimeter was constructed in a similar style to Patat \& Romaniello (2006). This simulates the observation of the individual o and e rays that would be observed for specific values of the total polarization $p$ and the polarization angle $\theta$ for a given a value of $S / N$. Different levels of gain and read-noise were applied to the o and e rays, in order to simulate the contribution of these factors to the corrections. The polarization induced by optical components preceding the analyzer, such as the telescope mirror, and polarization due to a non-ideal Wollaston prism were not included. For each simulation, the value of the difference of the $Q$ and $U$ corrections $\left(\epsilon_{Q}-\epsilon_{U}\right)$ was measured. The standard deviation of these values $\left(\Delta\left(\epsilon_{Q}-\epsilon_{U}\right)\right)$ about the mean value of $\epsilon_{Q}-\epsilon_{U}$ was used as a measure of the relative error. These terms permit the problem to be studied in terms of absolute deviations and the measurement error associated with the corrections.

For simple signal dominated observations, approaching ideal unvarying conditions, the behaviour of $\epsilon_{Q}-\epsilon_{U}$ is consistent with the analytical form presented in Sect. 3.1.

If there is a significant gain difference between the o and e rays, such that the ratio is $\gtrsim 10$, the determined Stokes parameters are dominated by signal measured in the ray with the larger gain. Importantly, the normalized flux difference is no longer applicable, and such observing conditions are unrealistic. Similarly, significant changes in the sky background $(\sim 100)$ between observations at each retarder plate angle can also induce deviations from $\epsilon_{Q}=\epsilon_{U}$, but this is also primarily due to the associated change in $S / N$ between observations. 


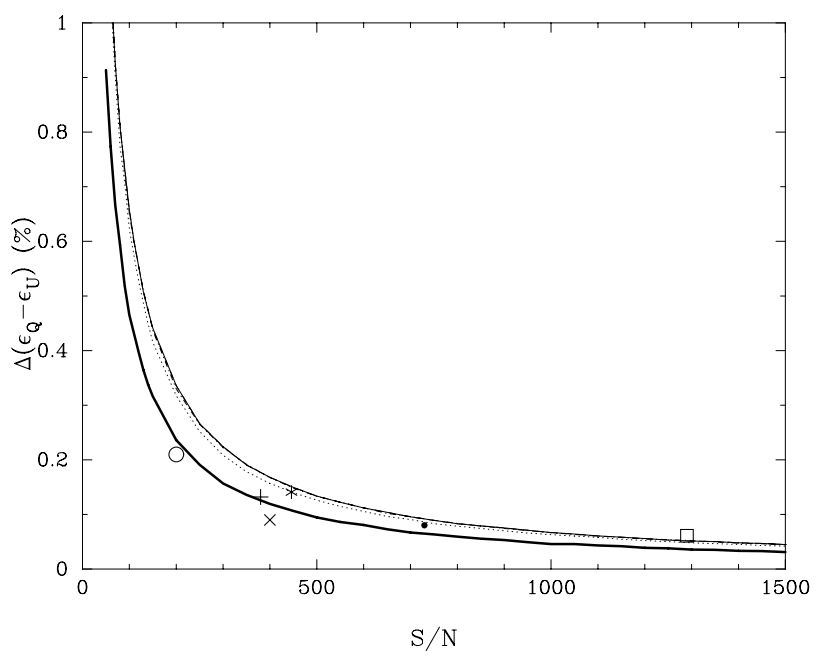

Fig. 3. The uncertainty (\%) on the difference between the corrections $\Delta\left(\epsilon_{Q}-\epsilon_{U}\right)$, of the $Q$ and $U$ Stokes parameters, as a function of the $S / N$. Calculations were conducted assuming the same parameters as FORS1 (Jehin et al. 2005), with $g=1.1$, between the $\mathrm{o}$ and e rays. All simulations were conducted with $\theta=60^{\circ}$. Simulations were conducted with $p=1 \%$ (light solid), $p=5 \%$ (dashed), $p=10 \%$ (dotdashed) and $p=50 \%$ (dotted). The error on the measured $Q$ Stokes parameters is shown as the heavy black line. Overlaid are measured values of $\Delta\left(\epsilon_{Q}-\epsilon_{U}\right)$ for six VLT FORS1 observations of SN 2001ig at 03 Jan. 2002 (口), HD 10038 at 07 Jul. $2003(\bullet)$, Vela 195 at 01 May 2005 (+), SN 2005hk at 23 Nov. 2005 (*) and SN 2006X at 09 Feb. 2006 (o) and 12 Feb. $2006(\times)$.

The value of $\epsilon_{Q}-\epsilon_{U}$ was calculated for varying values of $S / N$. Here the $S / N$ is calculated at each retarder plate position. In general, it was found that $\epsilon_{Q}-\epsilon_{U} \approx 0$, with $\Delta\left(\epsilon_{Q}-\epsilon_{U}\right)$ dependent on the level of $S / N$ in the individual observations, as shown as Fig. 3. Since the determination of $\epsilon_{Q}-\epsilon_{U}$ depends on 8 individual measurements, the o and e rays at the four retarder plate angles, the uncertainty $\Delta\left(\epsilon_{Q}-\epsilon_{U}\right)$ is $\sim \sqrt{2}$ larger than the uncertainty associated with the individual Stokes parameter. The quantity $\Delta\left(\epsilon_{Q}-\epsilon_{U}\right)$ is an estimate of the uncertainty of a Stokes parameter calculated from incomplete observations. Furthermore, the dependence of $\Delta\left(\epsilon_{Q}-\epsilon_{U}\right)$ on the polarization angle was also modelled, as shown in Fig. 4. As expected, the uncertainty on $\epsilon_{Q}-\epsilon_{U}$ is not directly dependent on polarization angle, although $\epsilon_{Q}-\epsilon_{U}$ is itself offset.

\section{Observations using VLT FORS1}

Spectropolarimetry observations of two SNe, one polarized standard star and one unpolarized standard star, acquired using the ESO VLT FORS1 instrument, were retrieved from the ESO archive $^{2}$, to determine if, under real observing circumstances, $\epsilon_{Q} \approx \epsilon_{U}$. These observations were of SN 2001ig at 03 Jan. 2002 (Maund et al. 2007a), the unpolarized standard HD 10038 at 07 Jul. 2003 (previously unpublished), the polarized standard Vela 195 at 01 May 2005 (Maund et al. 2007b), and SN 2005hk at 23 Nov. 2005 (Maund et al. 2008) ${ }^{3}$. These observations were conducted using the $300 \mathrm{~V}$ grism, and were re-binned to $30 \AA$. The observations were complete, with data at $N=4$ retarder plate angles, and were reduced in the standard manner as outlined by Maund et al. (2007b).

\footnotetext{
2 http://archive.eso.org

3 The reader is directed to the referenced publications for the exact nature of the polarization of each of these objects.
}

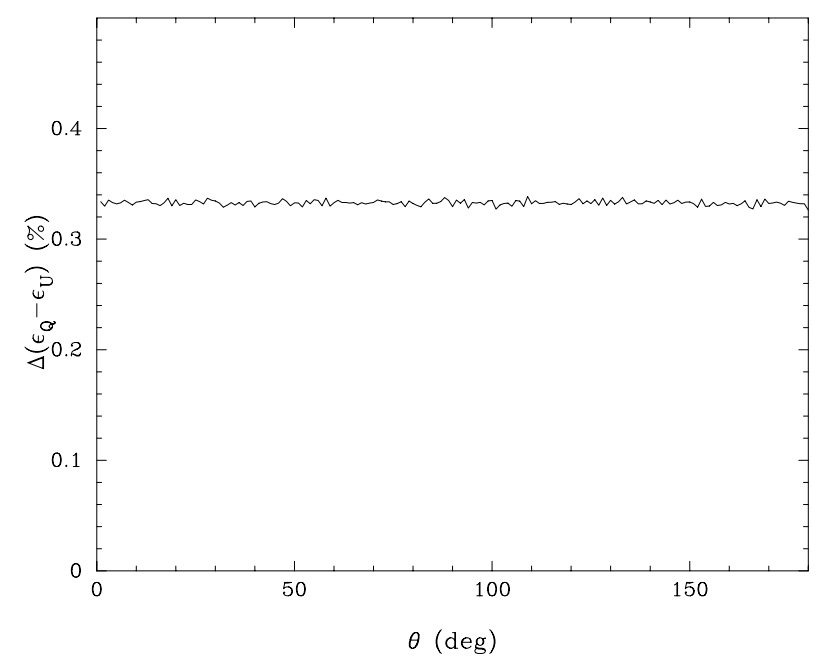

Fig. 4. The uncertainty ( $\%)$ of $\epsilon_{Q}-\epsilon_{U}$ as a function of the polarization angle. The calculation was made for $S / N=200$ and $p=1 \%$. The absolute value of $\epsilon_{Q}-\epsilon_{U}$ is a factor of 30 lower than the value of $\Delta\left(\epsilon_{Q}-\epsilon_{U}\right)$.

Observations of SNe were specifically chosen as: a) they represent the class of quick varying transients for which such a technique might be useful; and b) despite having diverse spectroscopic and polarimetric properties there has been a long term VLT FORS1 program since 2000 to observe these events using similar instrumental setups (namely the $300 \mathrm{~V}$ grism).

As each of these observations was complete, both $\epsilon_{Q}$ and $\epsilon_{U}$ could be determined; these are shown, as a function of wavelength, as Fig. 5. The corrections are observed to differ slightly between epochs. This implies that, just as new bias and flat calibration frames are required at each epoch, the instrumental signature correction, if it is to be applied to determine one Stokes parameter, needs to be determined from data acquired at the same epoch. The corrections are wavelength dependent, but not observed to be dependent on either the polarization or the spectroscopic properties of these objects. In instances of higher $S / N$ (such as Fig. 5a) the corrections are observed to be a smooth function of wavelength, with more variability observed for lower $S / N$ observations. Importantly, the largest deviations from $\epsilon_{Q}-\epsilon_{U}=0$ are observed for $\lambda>7000 \AA$, where there are a number of telluric absorption bands and the response of the FORS1 instrument is falling. For each of the four observations $\Delta\left(\epsilon_{Q}-\epsilon_{U}\right)$ was calculated over the wavelength range 5500-6500 $\AA$, to be representative of $\Delta\left(\epsilon_{Q}-\epsilon_{U}\right)$ at $6000 \AA$ at which the $S / N$ is quoted. For the given $S / N$ at $6000 \AA$ these values of $\Delta\left(\epsilon_{Q}-\epsilon_{U}\right)$ are plotted in Fig. 3 in comparison with the Monte Carlo model. The observed data agree well with the modelled dependence of $\Delta\left(\epsilon_{Q}-\epsilon_{U}\right)$ on the $S / N$ and polarization of the observations.

The observation of SN 2005hk (23 Nov. 2005; Fig. 5d) highlights the potential pitfalls of not observing a complete dataset. In the region $6500-7000 \AA$ there are a series of bad pixels which affected the o ray of the observation with the retarder plate at $45^{\circ}$, which could not be removed by bias subtraction or correction with a normalized flatfield. None of the observations at other retarder plate angles, for either the o and e ray, were affected. In this case, therefore, the calculation of $U$ by assuming $\epsilon_{U}=\epsilon_{Q}$ would lead to a serious error in the Stokes parameter without a substantial decrease in the $S / N$. The quality of the reduction at any wavelength, for a given $S / N$, can be directly measured by comparison of the $Q$ and $U$ instrumental signature corrections, 


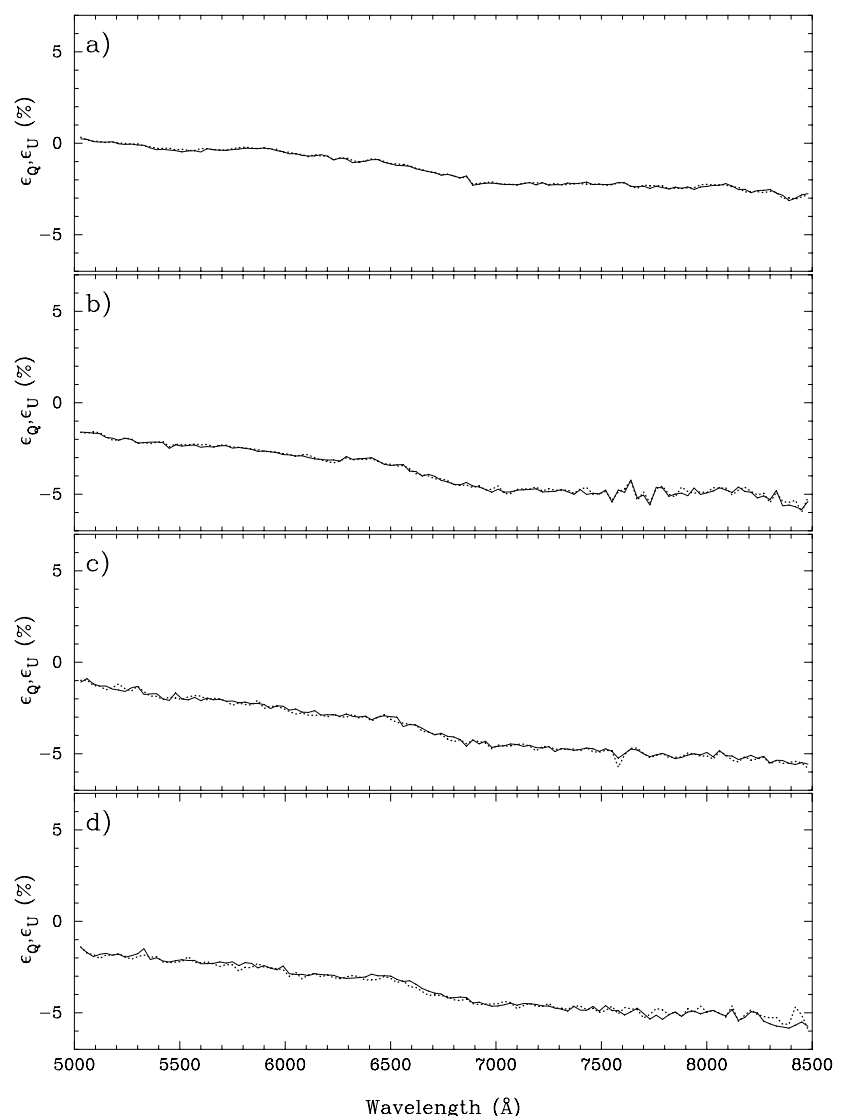

Fig. 5. $\epsilon_{Q}$ (solid line) and $\epsilon_{U}$ (dotted line), as a function of wavelength, for four complete FORS1 observations of: a) SN 2001ig at 03 Jan. 2002 $(S / N \sim 1290)$, b) unpolarized standard HD 10038 at 07 Jul. 2003 $(S / N \sim 730)$, c) polarized standard Vela 195 at 01 May $2005(S / N \sim$ $380)$, d) SN 2005hk at 23 Nov. 2005 ( $S / N \sim 446) . S / N$ values are given at $6000 \AA$. The data have be rebinned to $30 \AA$.

and the presence of such defects identified. Importantly, the $S / N$ is independent of the actual polarization, rather it is dependent on the total flux in both the o and e rays. The reduction may, therefore, be questioned if the absolute measured difference of $\epsilon_{Q}$ and $\epsilon_{U},\left|\epsilon_{Q}-\epsilon_{U}\right|_{\mathrm{m}}$, exceeds some multiple of the theoretically expected scatter, at a given $S / N$, e.g. $\left|\epsilon_{Q}-\epsilon_{U}\right|_{\mathrm{m}}>\left.3 \Delta\left(\epsilon_{Q}-\epsilon_{U}\right)\right|_{S / N}$.

In order to test the temporal stability of the absolute instrumental signature corrections, as a function of wavelength, a set of seven observations of the polarized standard star Vela 195 were retrieved from the ESO archive. These observations were acquired on 02 Oct. 2002, 01 Feb. 2003, 03 Feb. 2003, 07 May 2003, 30 Apr. 2005, 07 Jun. 2005 and 17 Mar. 2007, using an identical instrumental setup: the $300 \mathrm{~V}$ grism, no order separation filter and $2048 \times 500$ windowing of the CCD detector. There was not expected to be any significant effect due to detector windowing, but for consistency between the compared observations the windowing constraint was enforced. In order to directly compare the absolute values of the instrumental signature corrections at different epochs, the data were rebinned to $1000 \AA$ centred on 5000, 6000, 7000 and $8000 \AA$. The values for the instrumental signature correction at these epochs are shown as Fig. 6. The absolute values of the instrumental signature corrections vary between epochs. Additionally the wavelength dependence of the instrumental signature correction is also variable, with the gradient of the correction across the wavelength range (corresponding to the vertical distance between different wavelength points at the same epoch, in Fig. 6) changing. This

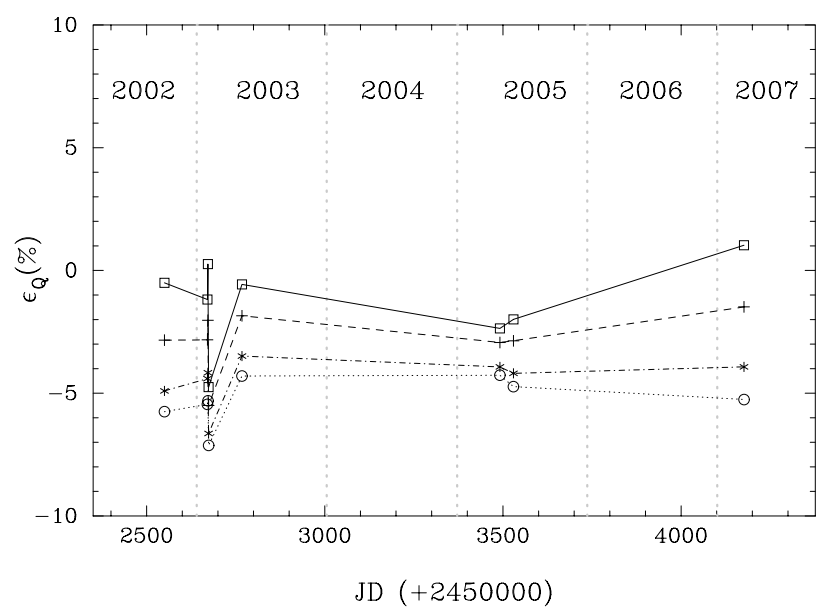

Fig. 6. The instrumental signature correction $\epsilon_{Q}$ for unfiltered VLT FORS1 300V PMOS observations of Vela 195 at 02 Oct. 2002, 01 Feb. 2003, 03 Feb. 2003, 07 May 2003, 30 Apr. 2005, 07 Jun. 2005, 17 Mar. 2007. The data shown are for $1000 \AA$ bins, centred on $5000 \AA$ (, solid line), $6000 \AA$ (+, dashed line), $7000 \AA$ (*, dot-dashed line), $8000 \AA$ ( o, dotted line).

Table 1. Linear spectropolarimetry data, of standard polarized stars, for selected VLT FORS1 grisms.

\begin{tabular}{llccc}
\hline \hline Grism & Object & Date & Program ID & Filters \\
\hline 150I & Hiltner 652 & 19 Sep. 1999 & 63.P-0002 & GG435 \\
300V & Hiltner 652 & 05 Apr. 2002 & 60.A-9203 & $\ldots$ \\
300I & BD 14 4922 & 23 Aug. 2002 & $60 . A-9203$ & OG590 \\
600B & HD 126593 & 22 Aug. 1999 & $63 . P-0074$ & $\ldots$ \\
600R & HD 126593 & 22 Aug. 1999 & 63.P-0074 & GG435 \\
600I & HD 126593 & 22 Aug. 1999 & 63.P-0074 & OG590 \\
\hline
\end{tabular}

confirms that instrumental signature corrections measured at different epochs cannot be used to reduce incomplete data acquired at other epochs and, hence, a minimum of $N=3$ observations are required.

The observed variability in the instrumental corrections may arise from evolution of the flatfield and of bias calibration frames, especially if the observed object spectra are not at the exact same location on the detector for each observation. Wavelength-dependent slit losses due to observations with the slits oriented with $\mathrm{PA}=0^{\circ}$, rather than the parallactic angle, may contribute to the varying gradients of the corrections. Importantly, despite the variability, $\epsilon_{Q}=\epsilon_{U}$ at each epoch.

The forms of the absolute values of $\epsilon_{Q}$ and $\epsilon_{U}$ are also dependent on the choice of grism. For the VLT FORS1, linear spectropolarimetry has been conducted with six grisms, for which observations of representative standard stars were selected (as listed in Table 1). The absolute forms of the corrections for observations with all six grisms used for spectropolarimetry are shown in Fig. 7. The $300 \mathrm{~V}$ grim is used with significantly higher frequency than the other grisms, such that the stability of the instrumental signature corrections for the other grisms cannot be tested with sufficient cadence or over a suitably long time frame as was done for $300 \mathrm{~V}$ above. The principal difference between the values of $\epsilon_{Q}$ and $\epsilon_{U}$ measured with different grisms is likely to arise from the response function of the detector for given wavelength dispersions. It is important that the values of $\epsilon_{Q}$ and $\epsilon_{U}$ for any particular grism cannot, in general, be extrapolated from those values measured with other grisms (except, potentially, in the cases of the $300 \mathrm{~V}$ and $150 \mathrm{I}$ grisms). 


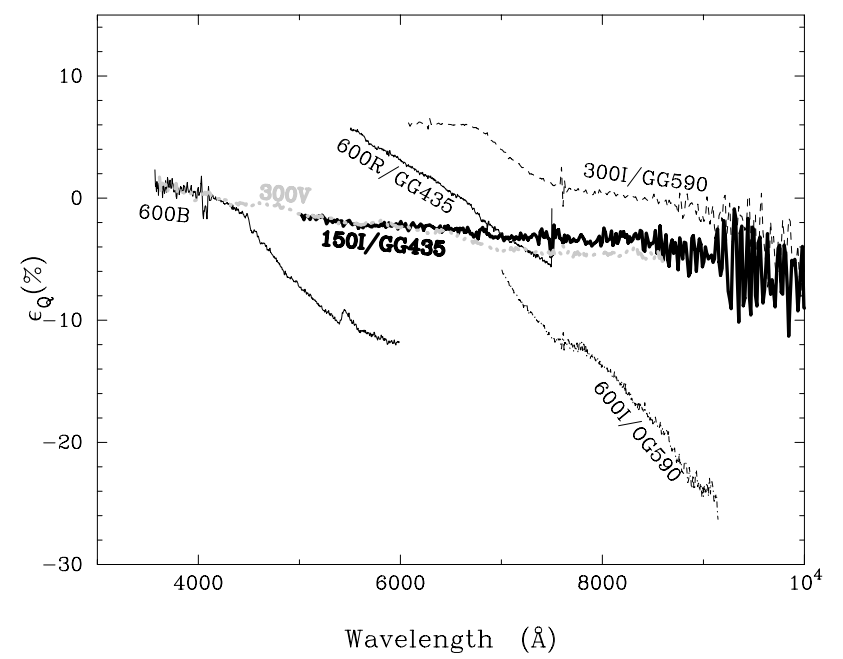

Fig. 7. The instrumental signature correction $\epsilon_{Q}$ for VLT FORS1 150I, 300I, 600B, 600R and 600I PMOS observations of polarized standard stars (listed in Table 1). The instrumental signature correction for the $300 \mathrm{~V}$ grism is shown as the grey line.

\section{Discussion and conclusions}

For spectropolarimetry where observations at $N=3$ retarder plate positions are available, the assumption that $\epsilon_{Q}=\epsilon_{U}$ can be employed to determine the additional Stokes parameter. This is a general property of dual beam-spectropolarimeters, which have negligible intrinsic instrumental polarization, and has been confirmed for VLT FORS1. If the Wollaston prism is non-ideal, the assumption of $\epsilon_{Q}=\epsilon_{U}$ is not true. This technique is still limited, as at least $N=3$ exposures in the standard observing sequence are required, although at a moderate expense of precision. Because the corrections are dependent on calibrations applied to the object data, the corrections calculated at different epochs are not strictly identical; although, in some cases they have been observed to be similar. The assumption $\epsilon_{Q}=\epsilon_{U}$ is not strictly true for observations of objects with linear polarization $\gtrsim 20 \%$. The uncertainty on $\epsilon_{Q}-\epsilon_{U}$ is dependent on the $S / N$ of the observations at each retarder plate angle and not the polarization of the object being observed. In some cases, and in the particular cases of some $\mathrm{SNe}$, this will permit reasonable spectropolarimetry in instances when it was not possible to acquire the complete set of observations.
In addition, the $\epsilon_{Q}-\epsilon_{U}$ parameter can also be used to identify defects and spurious polarization signatures in complete datasets. The $\Delta\left(\epsilon_{Q}-\epsilon_{U}\right)$ parameter can also be used to determine the quality of the data reduction procedure as applied to such datasets, as it is directly dependent on the $S / N$, and significant deviations from $\epsilon_{Q}-\epsilon_{U}=0$ would indicate the presence of defects in the data.

The instrumental signature corrections are dependent on wavelength, and are observed to be variable with time. Just as bias, flatfield and wavelength calibration observations are acquired for each epoch, the instrumental signature corrections should be determined at each epoch for each instrumental setup. Fortunately, this "additional" calibration information is derived from direct observations of target objects, requiring no additional observation time. The forms of $\epsilon_{Q}$ and $\epsilon_{U}$ are dependent on the choice of grism and the resulting dispersion across the detector.

Ultimately, the assumption of $\epsilon_{Q}-\epsilon_{U}=0$ is less than ideal, leading to larger degrees of uncertainty in the extra Stokes parameters by a factor of $\sim \sqrt{2}$ over parameters determined with $N=4$ observations. The use of these corrections does permit, however, the utilisation of data which would, ordinarily, remain unused (Maund et al. 2007c).

Acknowledgements. Based on observations made with ESO Telescopes at the Paranal Observatories under programmes 60.A-9203(A), 63.P-0002, 63.P-0074, 68.D-0571(A), 76.D-0177(A) and 76.D-178(A). The research of JRM is supported in part by NSF grant AST-0406740 and NASA grant NNG04GL00G. JRM thanks J. Craig Wheeler, Ferdinando Patat and Dietrich Baade for their useful comments, and also thanks Stefano Bagnulo, the referee, for his important suggestions.

\section{References}

Appenzeller, I., Fricke, K., Furtig, W., et al. 1998, The Messenger, 94, 1

Jehin, E., O’Brien, K., Kaufer, A. \& Hainaut, O., 2007, FORS1+2 User Manual, v81.1, ESO, Garching, VLT-MAN-ESO-13100-1543

Leonard, D. C., Filippenko, A. V., Ardila, D. R., \& Brotherton, M. S. 2001, ApJ, 553,861

Maund, J., Wheeler, J., Patat, F., Wang, L., Baade, D., \& Höflich, P. 2007a, ApJ, 671,1944

Maund, J., Wheeler, J., Patat, F., Baade, D., Wang, L., \& Höflich, P. 2007b, MNRAS, 381, 201

Maund, J., Wheeler, J., Patat, F., et al. 2007c, A\&A, 475, L1

Maund, J., Wheeler, J., Wang, L., et al. 2008, ApJ, in prep.

Patat, F., \& Romaniello, M. 2006, PASP, 118, 146 